\title{
Circumferential Measurement of Thoracic Wall Using a Standard Respiratory Belt
}

\author{
E. Rauvala, L. Jyrkinen, A. Koivula, K. Suominen, and O. Tervonen
}

\begin{abstract}
Respiratory gating during imaging to reduce imaging artifacts involves the gathering of image data only at the end of the respiratory cycle. This is commonly performed by a pneumatic respiratory belt to monitor thoracic wall motion during respiration. Such gating has been used for magnetic resonance, computerized tomographic, and nuclear medicine imaging. The goal of this study was to measure the performance of a standard belt used for gating imaging studies. The standard respiratory belt system provided with the Magnetom 42 SP MRI scanner (Siemens AG, Erlangen, Germany) was selected. The belt was connected to a microcontroller-based pressure measurement unit that was connected to the standard RS-232C serial port of a computer. The signal was compared with that of a strain gauge respiration transducer. The response of the system was tested in vitro both for isometric and isotonic loading. The data measured from the pneumatic beit was linear with different weights of 50 to 1,400 grams with a coefficient of determination $\left(R^{2}\right)$ of 0.999 . The system was linear for different amounts of stretching ( $R^{2}$ of 0.998 ) within the first $45 \mathrm{~mm}$, which is enough for normal breathing. In vivo the pneumatic system seemed more accurate in measuring the constant stretching in apnea than the strain gauge respiratory belt. The results show that it is possible to use a standard pneumatic belt for accurate measurement of thoracic wall movement during imaging and for other purposes as well.
\end{abstract}

Copyright 1996 by W.B. Saunders Company

KEY WORDS: respiratory, thoracic, circumferential, measurement, belt, gating, computers, radiology, magnetic resonance imaging (MRI).

$\mathbf{T}$ HE MOVEMENT of thoracic and abdominal organs during the respiratory cycle causes artifacts in imaging these regions. It also makes interventional procedures more difficult because of the uncertainty of the exact location of organs. Respiratory gating has been used for magnetic resonance (MR), computerized tomographic (CT), and nuclear medicine (NM) imaging.

From the Department of Diagnostic Radiology and the Department of Clinical Neurophysiology, University Central Hospital of Oulu, Oulu, Finland.

Supported by The Finnish Center for Technological Advance. ment (TEKES)

Address reprint requests to $O$. Tervonen, $M D$, Radiologian klinikka, OYKS, 90220 Oulu, Finland.

Copyright 1996 by W.B. Saunders Company

0897-1889/96/0902-0004\$3.00/0
There are several methods for minimizing the imaging artifacts caused by respiration. One method is to allow gathering of imaging data from either end of the respiratory cycle where the thoracic and abdominal organ movements are at their minimum. This is called respiratory gating, and it is commonly performed by a respiratory belt using a pneumatic capsule for monitoring thoracic wall motion during a respiratory cycle. The belt is attached around the chest or the abdomen. When connected to a MR scanner by a tube, measurements cannot be made outside of the MR scanner facility. Estimation of upper abdominal organ location also requires data on the phase of respiration.

The purpose of the present study was to test the accuracy of the data provided by the measurement system applying the respiratory belt and to compare the system with a strain gauge respiratory transducer system. No published articles on the accuracy of a pneumatic belt for respiratory gating compared to other techniques were found in a MEDLINE CD-ROM (EBSCO Publishing, Peabody, MA) search of the literature.

\section{MATERIALS AND METHODS}

The standard respiratory belt system provided by the MRI scanner (Magnetom 42 SP; Siemens AG, Erlangen,

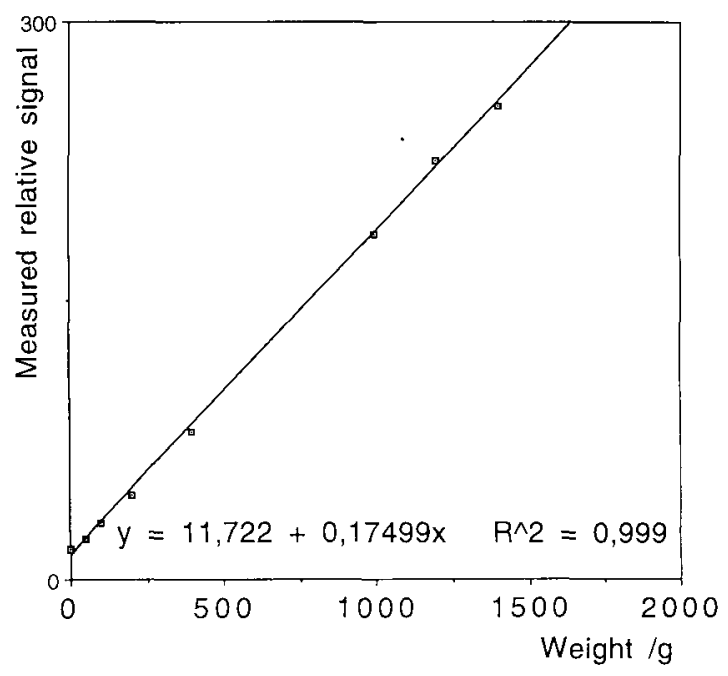

Fig 1. Isotonic loading of the belt. 


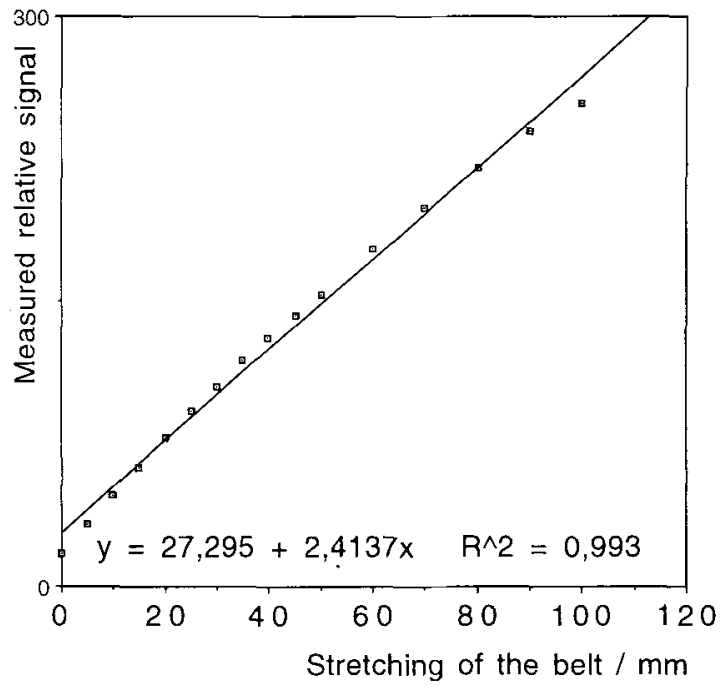

Fig 2. Isometric loading of the belt.

Germany) consists of a nonstretchable belt and a stretchable rubber capsule that is connected to the MRI scanner. This system was connected to a microcontroller-based pressure measurement unit (MPMU) with a rubber tube approximately $440 \mathrm{~cm}$ in length with one connector in the middle, the external diameter of the tube being $6.5 \mathrm{~mm}$ and the internal $3 \mathrm{~mm}$ making the internal volume approximately $30 \mathrm{~mL}$. The unit was constructed from a pressure sensor (Motorola MPX2100D, Phoenix, AZ), a preamplifier, and a controller (Motorola M68HC11) (see Fig 6).

The amplitude of the measured signal is amplified by a factor of 700 and the signal is then digitized by the microcontroller's internal A/D-converter. The MPMU was connected to the standard RS-232C serial port of a computer. The computer controls the MPMU measurements, and it in turn transmits the measured data to the computer.

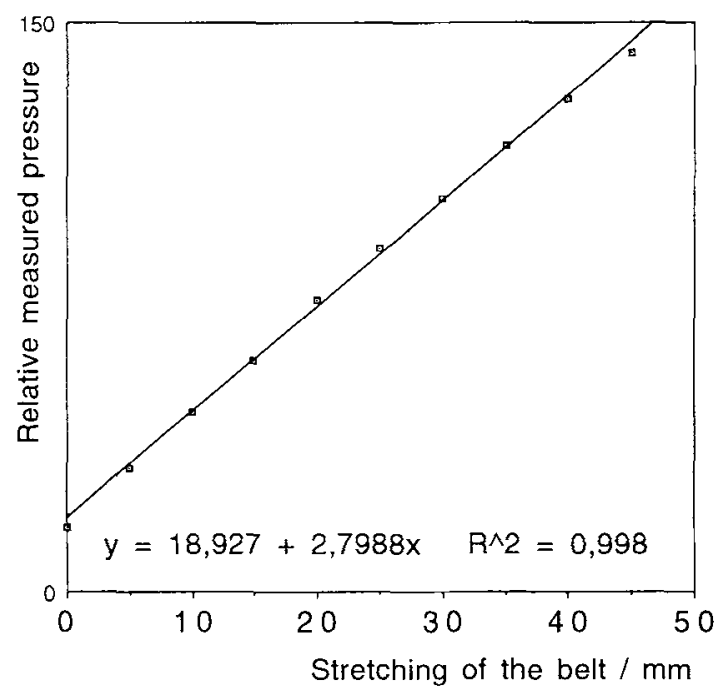

Fig 3. Isometric loading; short range.

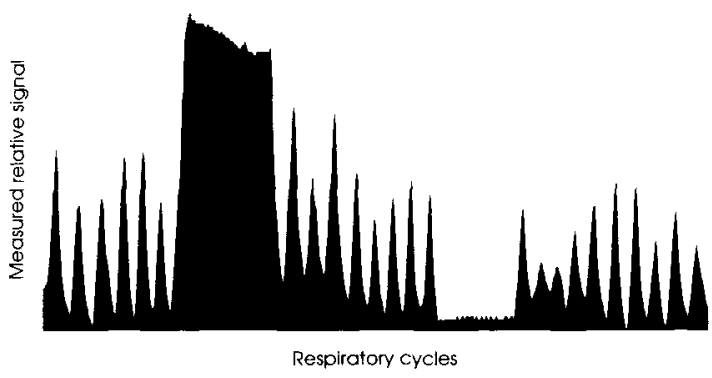

Fig 4. Respiratory cycles by pneumatic belt.

The signal provided by this system was compared with that of a strain gauge respiration transducer (Lectromed respiration transducer 4320; Lectromed Ltd, Jersey, UK) used in the department of clinical neurophysiology for nonimaging testing (sleep apnea diagnosis).

The response of the system was tested in vitro both for isometric and isotonic loading. Both methods were used to detect the possible nonlinearity of the pneumatic capsule's mechanical stretching. The measurement unit's isometric linearity was tested using different constant weights of 50 to $1,400 \mathrm{~g}$ hanging from the belt. The response of the system for isotonic loading was evaluated by constant stretching of $5 \mathrm{~mm}$ to $10 \mathrm{~cm}$ of the pneumatic capsule.

The system was evaluated in vivo by measuring the signal simultaneously using both the strain gauge and the pneumatic respiratory belt attached around the thorax below mammillary level of one healthy male volunteer in sitting position.

The measured data was analyzed using statistical and graphical software (Cricket Graph 1.3.1; Cricket Software, Malvern, PA and Excel 4.0; Microsoft, Seattle, WA).

\section{RESULTS}

The data measured from the pneumatic capsule was practically linear with different weights with a coefficient of determination $\left(\mathrm{R}^{2}\right)$ of 0.999 (Fig 1).

In the case of different amounts of stretching the system was linear up to about $45 \mathrm{~mm}$, above which the measured signal started to relatively diminish. Within the first $45 \mathrm{~mm}$ the $\mathrm{R}^{2}$ was

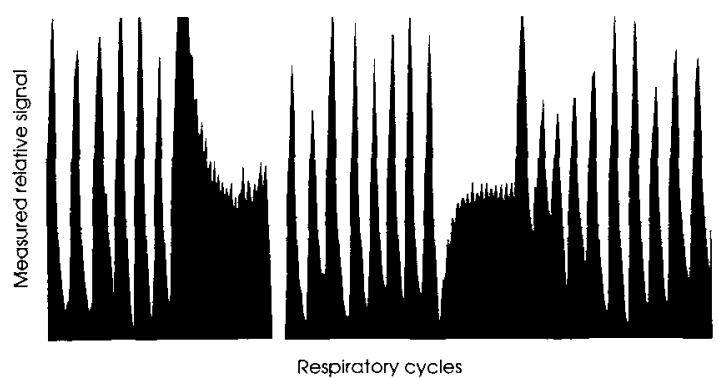

Fig 5. Respiratory cycles by strain gauge belt. 


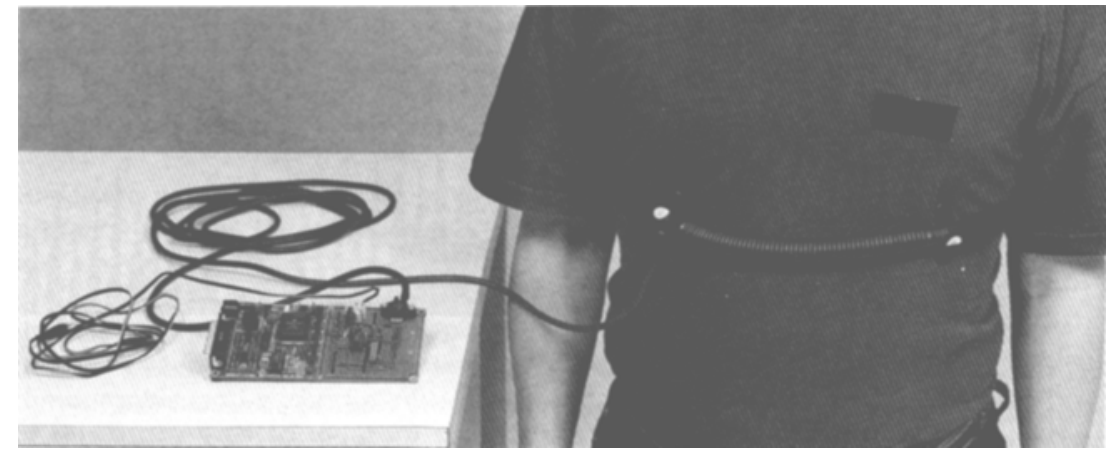

Fig 6. Respiratory belt connected to the measurement unit.
0.998. In the total range $100 \mathrm{~mm}$, the $\mathrm{R}^{2}$ was reduced to 0.993 (Figs 2 and 3).

The strain gauge respiratory transducer's amplifier has a band-pass filter, which causes the signal to approach a base level according to a time constant. Therefore, its accuracy could not be evaluated using similar methods.

The accuracy of the strain gauge system was also confirmed by comparing a simultaneous graphical presentation of the strain gauge data during a volunteer's breathing. The two systems were compared, and it was concluded that with both systems it was possible to recognize all phases of the respiratory cycles without difficulty and without false positives. However, the pneumatic system seemed more accurate in measuring the constant stretching in apnea because the strain gauge respiratory transducer's signal reaches base level within seconds. (Figs 4 and 5)

\section{DISCUSSION}

The evaluation of diaphragmatic and abdominal organ movement during respiration requires information of many properties of respiratory mechanics. (1) Thoracic wall movement has to be measured with sufficient accuracy. (2) The relationships of diaphragmatic and thoracic wall movement in a respiratory cycle and the temporal properties of these relationships must be estimated. (3) The organs of the upper abdomen also undergo changes in conformation and position. Respiratory belts wrapped around the chest are routinely used for artifact reduction, either for gating or for reordering of data. The accuracy of these belts is poorly documented in the literature, but the results from the present study show that accurate circumferential measurement of thoracic wall movement can be obtained by using the respiratory belt. The linear range of the tested system of circa $45 \mathrm{~mm}$ is enough for normal respiration, and the linearity in the range of $10 \mathrm{~cm}$ is relatively good also. With this system it is also possible to get absolute data about the phase of thoracic movement. The other two aspects of respiratory organ movement still remain to be researched.

The system configuration including the standard RS 232 serial port used in this study makes it easy to connect the system to almost any computer (Fig 6). In turn this means that this standard belt system can be used in settings outside of the MR suite as the pneumatic connection is not required. The relation between stretching of the belt and measured data is linear. Its accuracy also seems good enough for nonimaging applications including the diagnosis of sleep apnea.

The results show that it is possible to use the standard pneumatic belt for accurate measurement of thoracic wall movement during the respiratory cycle. This system is suitable for gating MR, CT, ultrasonography, and NM imaging studies. 